\title{
New Insights into the Diversity of the Genus Faecalibacterium
}

\author{
Leandro Benevides $^{1,2}$, Sriti Burman ${ }^{3}$, Rebeca Martin ${ }^{2}$, Véronique Robert ${ }^{2}$, \\ Muriel Thomas ${ }^{2}$, Sylvie Miquel ${ }^{2,4}$, Florian Chain ${ }^{2}$, Harry Sokol $^{2,5,6}$, \\ Luis G. Bermudez-Humaran ${ }^{2}$, Mark Morrison $^{3}$, Philippe Langella $^{2}$, Vasco A. Azevedo ${ }^{1}$, \\ Jean-Marc Chatel $^{2 \dagger}$ and Siomar Soares ${ }^{7 *}$
}

${ }^{1}$ Department of General Biology, Federal University of Minas Gerais, Belo Horizonte, Brazil, ${ }^{2}$ Commensals and Probiotics-Host Interactions Laboratory, Micalis Institute, Institut National de la Recherche Agronomique, AgroParisTech, Université Paris-Saclay, Jouy-en-Josas, France, ${ }^{3}$ Faculty of Medicine, Translational Research Institute, University of Queensland Diamantina Institute, University of Queensland, St. Lucia, QLD, Australia, ${ }^{4}$ UMR 6023 Laboratoire

OPEN ACCESS

Edited by:

Geanncarlo Lugo-Villarino,

UMR5089 Institut de Pharmacologie et de Biologie Structurale (IPBS),

France

Reviewed by:

Clara G. De Los Reyes-Gavilan, Consejo Superior de Investigaciones Cientificas (CSIC), Spain

Ulisses Padua Pereira, Universidade Estadual de Londrina,

Brazil

${ }^{*}$ Correspondence: Siomar Soares siomars@gmail.com

${ }^{\dagger}$ These authors have contributed equally to this work.

Specialty section:

This article was submitted to

Microbial Immunology,

a section of the journal

Frontiers in Microbiology

Received: 03 August 2017 Accepted: 05 September 2017 Published: 22 September 2017

Citation:

Benevides L, Burman S, Martin R, Robert $V$, Thomas M, Miquel $S$, Chain F, Sokol $H$, Bermudez-Humaran LG, Morrison M,

Langella P, Azevedo VA, Chatel J-M and Soares S (2017) New Insights into the Diversity of the Genus Faecalibacterium.

Front. Microbiol. 8:1790. doi: 10.3389/fmicb.2017.01790
Microorganismes: Génome et Environnement, Centre National de la Recherche Scientifique, Université Clermont Auvergne, Clermont-Ferrand, France, ${ }^{5}$ Faculté de Médecine Saint-Antoine, Institut National de la Santé et de la Recherche Médicale U1157/UMR7203, AVENIR Team Gut Microbiota and Immunity Equipe de Recherche Labélisée, Université Pierre et Marie Curie, Paris, France, ${ }^{6}$ Service de Gastroentérologie, Hôpital Saint-Antoine, Assistance Publique-Hôpitaux de Paris, Paris, France, ${ }^{7}$ Department of Immunology, Microbiology and Parasitology, Institute of Biological Sciences and Natural Sciences, Federal University of Triângulo Mineiro, Uberaba, Brazil

Faecalibacterium prausnitzii is a commensal bacterium, ubiquitous in the gastrointestinal tracts of animals and humans. This species is a functionally important member of the microbiota and studies suggest it has an impact on the physiology and health of the host. F. prausnitzii is the only identified species in the genus Faecalibacterium, but a recent study clustered strains of this species in two different phylogroups. Here, we propose the existence of distinct species in this genus through the use of comparative genomics. Briefly, we performed analyses of 16S rRNA gene phylogeny, phylogenomics, whole genome Multi-Locus Sequence Typing (wgMLST), Average Nucleotide Identity (ANI), gene synteny, and pangenome to better elucidate the phylogenetic relationships among strains of Faecalibacterium. For this, we used 12 newly sequenced, assembled, and curated genomes of $F$. prausnitzii, which were isolated from feces of healthy volunteers from France and Australia, and combined these with published data from 5 strains downloaded from public databases. The phylogenetic analysis of the 16S rRNA sequences, together with the wgMLST profiles and a phylogenomic tree based on comparisons of genome similarity, all supported the clustering of Faecalibacterium strains in different genospecies. Additionally, the global analysis of gene synteny among all strains showed a highly fragmented profile, whereas the intra-cluster analyses revealed larger and more conserved collinear blocks. Finally, ANI analysis substantiated the presence of three distinct clusters - A, B, and C-composed of five, four, and four strains, respectively. The pangenome analysis of each cluster corroborated the classification of these clusters into three distinct species, each containing less variability than that found within the global pangenome of all strains. Here, we propose that comparison of pangenome subsets and their associated $\alpha$ values may be used as an alternative approach, together with ANI, in the in silico classification of new species. Altogether, 
our results provide evidence not only for the reconsideration of the phylogenetic and genomic relatedness among strains currently assigned to F. prausnitzii, but also the need for lineage (strain-based) differentiation of this taxon to better define how specific members might be associated with positive or negative host interactions.

Keywords: Faecalibacterium prausnitzii, genome sequencing, 16S rRNA gene phylogeny, phylogenomic analysis, Average Nucleotide Identity, gene synteny, pangenome, new species

\section{INTRODUCTION}

Members of genus Faecalibacterium are commensal bacteria, ubiquitous in the gastrointestinal tracts of animals and humans. Within the human colon, this taxon is the main member of the Clostridium leptum cluster, and comprises the second-most common representative in fecal samples, after Clostridium coccoides (Tap et al., 2009; Walker et al., 2011). The first characterized isolates were classified as Fusobacterium prausnitzii, but its close relationship with other members of the C. leptum cluster (phylum Firmicutes, class Clostridia, family Ruminococcaceae) was later established through analysis of the 16S rRNA gene of different strains found in humans (ATCC 27766 and ATCC 27768) (Wang et al., 1996; Duncan et al., 2002). The relative abundance of $F$. prausnitzii in vertebrate animals other than humans, such as pigs (Castillo et al., 2007), mice (Nava and Stappenbeck, 2011), calves (Oikonomou et al., 2013), and chickens (Scupham, 2007), suggests that the species is a functionally important member of the microbiota and likely has an impact on the physiology and health of the host. In that context, changes in the abundance of $F$. prausnitzii have been widely described in various intestinal and metabolic diseases in humans, such as colorectal cancer (CRC), Crohn's disease (CD), and ulcerative colitis (UC) (Sokol et al., 2008; RajilićStojanović et al., 2011; Miquel et al., 2013). Due to its ubiquity and immunomodulatory properties, some studies suggest that F. prausnitzii is an indicator of, and an active contributor to, intestinal health and the maintenance of gut homeostasis (Sokol et al., 2008; Miquel et al., 2013, 2014). Despite its relevance in the human gut ecosystem, little is known about the diversity of $F$. prausnitzii (Miquel et al., 2014) and only a few studies have examined isolated strains and used functional approaches (Duncan et al., 2002; Lopez-Siles et al., 2012). To better understand the biodiversity and beneficial effect of this species, it is essential to increase our knowledge of several cultured strains and their genomes.

Recent studies, based on 16S rRNA sequence analyses, have suggested the existence of two phylogroups within this species (Duncan et al., 2002; Lopez-Siles et al., 2012, 2017). Here we present a new phylogenetic and comparative study of five sequenced genomes of $F$. prausnitzii available in public databases, combined with twelve new genome sequences isolated from healthy volunteers in Europe and Australia. The phylogenetic relationships among these isolates of $F$. prausnitzii were compared, and pangenomic analyses provided us a more global view of the genomic diversity across these strains. These data will enable new insights into the contributions of genus Faecalibacterium to gut function.

\section{METHODS}

\section{Genome Sequencing, Assembly, and Annotation}

The genomes used in this study are presented in Table 1. The genome data of five different $F$. prausnitzii strains were retrieved from the PATRIC public database. These were combined with genome data from ten newly isolated $F$. prausnitzii strains recovered from stool samples of healthy European volunteers (Martín et al., 2017), as well as two newly isolated F. prausnitzii strains recovered from stool samples of healthy Australian subjects (following the guidelines of the University of Queensland Human Research Ethics Committee \#2015000775). In Europe, the ten new genomes were sequenced by GATC Biotech Company using the Illumina HiSeq2500 platform; the genomes from the Australian isolates were sequenced using the Illumina NextSeq platform at the Australian Centre for Ecogenomics (www.ecogenomic.org). The genome of the wild-type strain $F$. prausnitzii A2-165 (F. prausnitzii_A2165_PacBio) was sequenced using PacBio single-molecule realtime (SMRT) technology on an RS system (Pacific Bioscience) and assembled by the GATC Biotech Company. The quality of the sequenced reads was checked with FastQC software (http:// www.bioinformatics.babraham.ac.uk/projects/fastqc/). All of the genomes (except for wild-type strain A2-165) were assembled using a de novo strategy with SPAdes software, v3.8.0. The quality of the assemblies was evaluated using QUAST software (Gurevich et al., 2013) and all genomes were subjected to automated functional annotation using the RAST server (Aziz et al., 2008).

\section{Phylogeny}

Phylogenetic analyses were performed on 16S rRNA sequences, whole genome sequences, and the results of whole-genome Multi-Locus Sequence Typing (wgMLST). For the first analysis, the 16S rRNA sequence from the genome of $F$. prausnitzii_A2165_PacBio was used to perform a BLASTn search in the NCBI database for all sequences belonging to the genus Faecalibacterium. The sequence results with more than $82 \%$ coverage and $92 \%$ identity were collected, and the $16 \mathrm{~S}$ rRNA gene sequence from Subdoligranulum variabile BI-114 was included as an outgroup. Sequences were then aligned using the multiple sequence alignment tool CLUSTALW (Thompson et al., 1994) integrated in MEGA7 software (Kumar et al., 2016). After that, the most appropriate evolutionary model was defined and evolutionary history was inferred using the maximumlikelihood (ML) criterion, based on the Kimura 2-parameter model (Kimura, 1980), with 1,000 bootstrap replicates. 
TABLE 1 | Genomic features of F. prausnitzii genomes.

\begin{tabular}{|c|c|c|c|c|c|c|c|}
\hline Genome name & $\begin{array}{c}\text { PATRIC } \\
\text { genome ID }\end{array}$ & $\begin{array}{l}\text { Genbank } \\
\text { accessions }\end{array}$ & Isolation country & Sequences & $\begin{array}{l}\text { Genome } \\
\text { length }\end{array}$ & $\begin{array}{c}\text { GC } \\
\text { content }\end{array}$ & $\begin{array}{c}\text { PATRIC } \\
\text { CDS }\end{array}$ \\
\hline Faecalibacterium prausnitzii M21/2 & 411485.10 & ABED00000000 & United Kingdom & 25 & $3,125,761$ & 56.3 & 2,776 \\
\hline Faecalibacterium prausnitzii SL3/3 & 657322.3 & FP929046 & United Kingdom & 1 & $3,214,418$ & 54.81 & 3,052 \\
\hline Faecalibacterium prausnitzii L2-6 & 718252.3 & FP929045 & United Kingdom & 1 & $3,321,367$ & 55.57 & 3,232 \\
\hline Faecalibacterium cF. prausnitzii KLE1255 & 748224.3 & AECU00000000 & USA & 139 & $2,907,000$ & 56.27 & 2,783 \\
\hline Faecalibacterium prausnitzii AHMP_21 & 853.123 & NOUV00000000 & Australia & 85 & $3,019,317$ & 57.36 & 3,201 \\
\hline Faecalibacterium prausnitzii HMI_19 & 853.124 & NOUW00000000 & Australia & 63 & $2,879,169$ & 56.82 & 2,933 \\
\hline Faecalibacterium prausnitzii CNCM_4540 & 853.62 & NMTQ00000000 & France & 48 & $3,043,568$ & 55.7 & 3,206 \\
\hline Faecalibacterium prausnitzii CNCM_4541 & 853.63 & NMTR00000000 & France & 78 & 2,822,838 & 58.11 & 2,825 \\
\hline Faecalibacterium prausnitzii CNCM_4542 & 853.64 & NMTS00000000 & France & 106 & $2,914,466$ & 55.83 & 3,071 \\
\hline Faecalibacterium prausnitzii CNCM_4543 & 853.65 & NMTT00000000 & France & 22 & $3,080,452$ & 56.2 & 3,223 \\
\hline Faecalibacterium prausnitzii CNCM_4544 & 853.66 & NMTU00000000 & France & 71 & $2,808,526$ & 55.98 & 2,907 \\
\hline Faecalibacterium prausnitzii CNCM_4546 & 853.67 & NMTV00000000 & France & 244 & $3,422,520$ & 54.88 & 3,611 \\
\hline Faecalibacterium prausnitzii CNCM_4573 & 853.68 & NMTW00000000 & France & 83 & $3,275,249$ & 55.9 & 3,479 \\
\hline Faecalibacterium prausnitzii CNCM_4574 & 853.69 & NMTX00000000 & France & 38 & 3,088,985 & 56.26 & 3,249 \\
\hline Faecalibacterium prausnitzii CNCM_4575 & 853.70 & NMTY00000000 & France & 37 & $3,006,602$ & 57.51 & 3,077 \\
\hline Faecalibacterium prausnitzii CNCM_4644 & 853.71 & NMTZ00000000 & France & 36 & $2,915,240$ & 56.37 & 3,019 \\
\hline Faecalibacterium prausnitzii A2-165_PacBio & 853.73 & CP022479 & France & 1 & $3,110,044$ & 56.33 & 3,231 \\
\hline
\end{tabular}

The phylogenomic analysis was performed using Gegenees software (Agren et al., 2012), which calculated the percentage of similarity among the genomes of all strains. Before calculating similarity scores, we used the BLASTn alignment method, with a sequence fragmentation length of $200 \mathrm{bp}$ and a step size of $100 \mathrm{bp}$. The input files for Gegenees contained the complete genomes in ".fna" format and the resulting similarity matrix was exported in ".nexus" format for phylogenomic analysis using SplitsTree4 software (Huson, 2005). The equal angle method was used to construct the phylogenetic network, which was plotted with NeighborNet.

A wgMLST analysis was performed using the Build_wgMLSTtree module in the PGAdb-builder web service tool (Liu et al., 2016). The 17 genome sequences were compared with the resulting PGAdb profile using BLASTn, with filters of $80 \%$ coverage and $90 \%$ identity.

\section{Average Nucleotide Identity}

We also performed an Average Nucleotide Identity (ANI) analysis using the whole-genome sequences. ANI represents a mean of identity/similarity values between homologous genomic regions shared by two genomes. It is generally accepted that ANI values of 95-96\% equate to a DNA-DNA hybridization (DDH) value of $70 \%$, and can be used as a threshold for species delineation (Konstantinidis and Tiedje, 2005; Kim et al., 2014).

\section{Gene Synteny Analysis}

The progressiveMauve option from the Mauve package (Darling et al., 2004) was used with default parameters to perform orthology comparisons and to evaluate gene synteny among the genomes of $F$. prausnitzii. This genome comparison method also predicts syntenic blocks, which reveal the rearrangement events among the genomes (Darling et al., 2004). This analysis was performed using four different datasets: first, using all 17 genomes, and then using the genome subsets of each of the three groups that resulted from ANI analysis.

\section{Pangenome Calculation}

The software program OrthoMCL (Li et al., 2003) was used first to define the cluster of orthologous genes and then, the commonly shared and species-specific genes of all the strains and subgroups. The amino-acid sequences from all coding DNA sequences (CDSs) in each genome were first used in an all-vs.all BLASTp analysis with an $e$-value of $10^{-6}$; the sequences were then clustered using the MCL algorithm. The CDSs observed in all strains were considered to comprise the core genome, while the CDSs harbored by only one strain were considered to be singleton genes.

To calculate pangenome development, we applied Heap's Law, with the formula $n=k^{*} N^{-\alpha}$, where $n$ is the expected number of genes for a given number of genomes, $N$ is the number of genomes, and the other terms are constants defined to fit the specific curve. According to Heap's law, a value of $\alpha \leq 1$ is representative of an open pangenome; this means that each added genome will contribute some new genes and the pangenome will increase. Instead, an $\alpha$ value $>1$ represents a closed pangenome, in which the addition of new genomes will not significantly affect the size of the pangenome. The extrapolations of the curves of the core genome and singletons were calculated using the leastsquares fit of the exponential regression decay of the mean values, as represented by the formula $n=\mathrm{k}^{*} \exp [-\mathrm{x} / \mathrm{t}]+\operatorname{tg}(\theta)$, where $\mathrm{n}$ is the expected subset of genes for $\mathrm{x}$ number of genomes, exp is Euler's number, and the other terms are constants defined to fit 


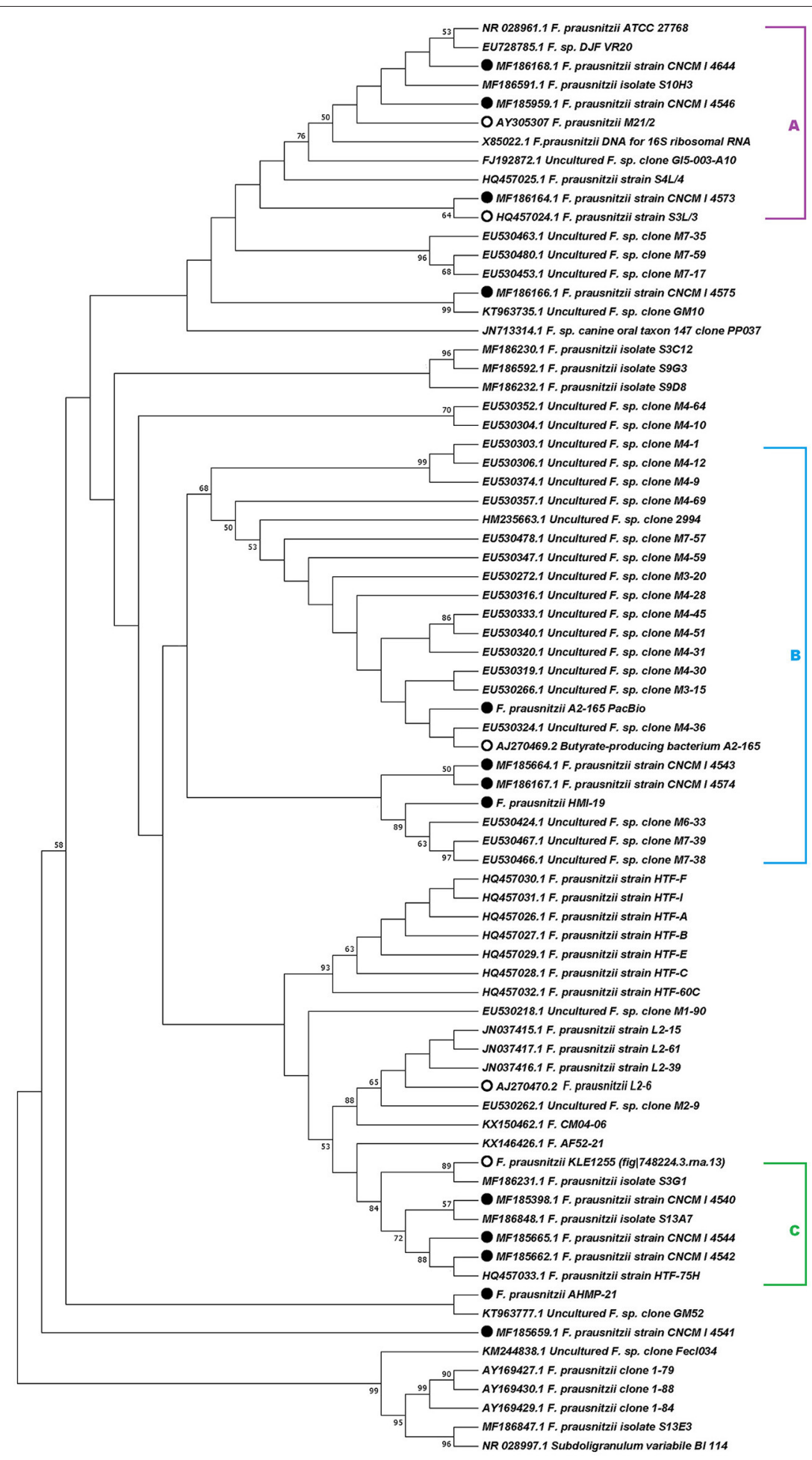

FIGURE 1 | Phylogenetic analysis based on 16 S rRNA gene sequences. Evolutionary history was inferred using the maximum-likelihood method based on the Kimura 2-parameter model (Kimura, 1980). The topology of the tree with the highest log likelihood $(-3,562.92)$ is shown. The percentage of trees in which the associated taxa clustered together is shown next to the branches. A discrete Gamma distribution was used to model evolutionary rate differences among sites [5 categories (+ $\mathrm{G}$, parameter $=0.1122)]$. The tree is drawn to scale, with branch lengths measured as the number of substitutions per site. The analysis involved 76 nucleotide sequences. All positions containing gaps and missing data were eliminated. The bootstrap analysis was performed with 1,000 replicates. Evolutionary analyses were conducted in MEGA7 (Kumar et al., 2016). Accession numbers of 16S rRNA sequences are given in parentheses. Filled circles indicate the strains newly sequenced for this study and open circles indicate the strains retrieved from PATRIC for genomic analysis. 
the specific curve. The formula used to calculate the extrapolated curves of core and singleton genes can be used to predict the final number of CDSs if we consider a high number of genomes. In this formula, the value of $\operatorname{tg}(\theta)$ represents the convergence value of the size of the core genome or the number of new genes (singletons).

\section{RESULTS}

\section{General Features}

The number of contigs in the draft genomes varied from 22 to 244 . The genome lengths varied by $613,994 \mathrm{bp}$ in size. The GC content varied from $54.81 \%$ (F. prausnitzii SL3/3) to $58.11 \%$
(F. prausnitzii CNCM_4541) and the number of predicted CDSs varied from 2,776 to 3,611 (Table 1).

\section{Phylogeny}

The phylogenetic analysis of 16S rRNA sequences revealed that the genospecies of Faecalibacterium can be clustered into different groups. Specifically, the 16S rRNA gene sequences from the new French genomes grouped into clusters A, B, and C (Figure 1), as previously proposed by Martín et al. (2017). The 16S rRNA gene sequence from one Australian isolate, F. prausnitzii HMI-19, clustered with sequences in group B, whereas the other Australian sequence (F. prausnitzii

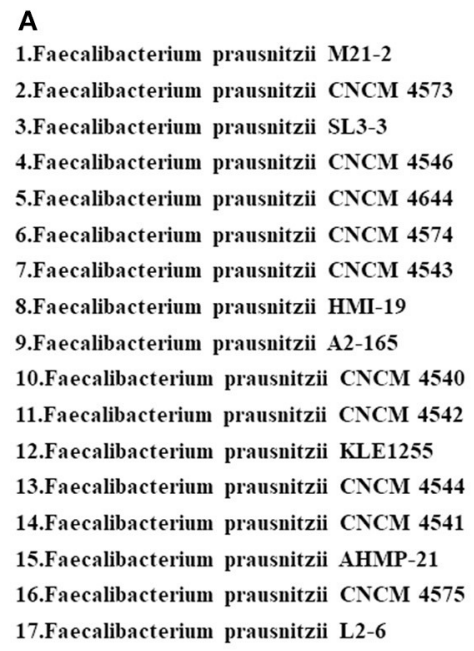

B

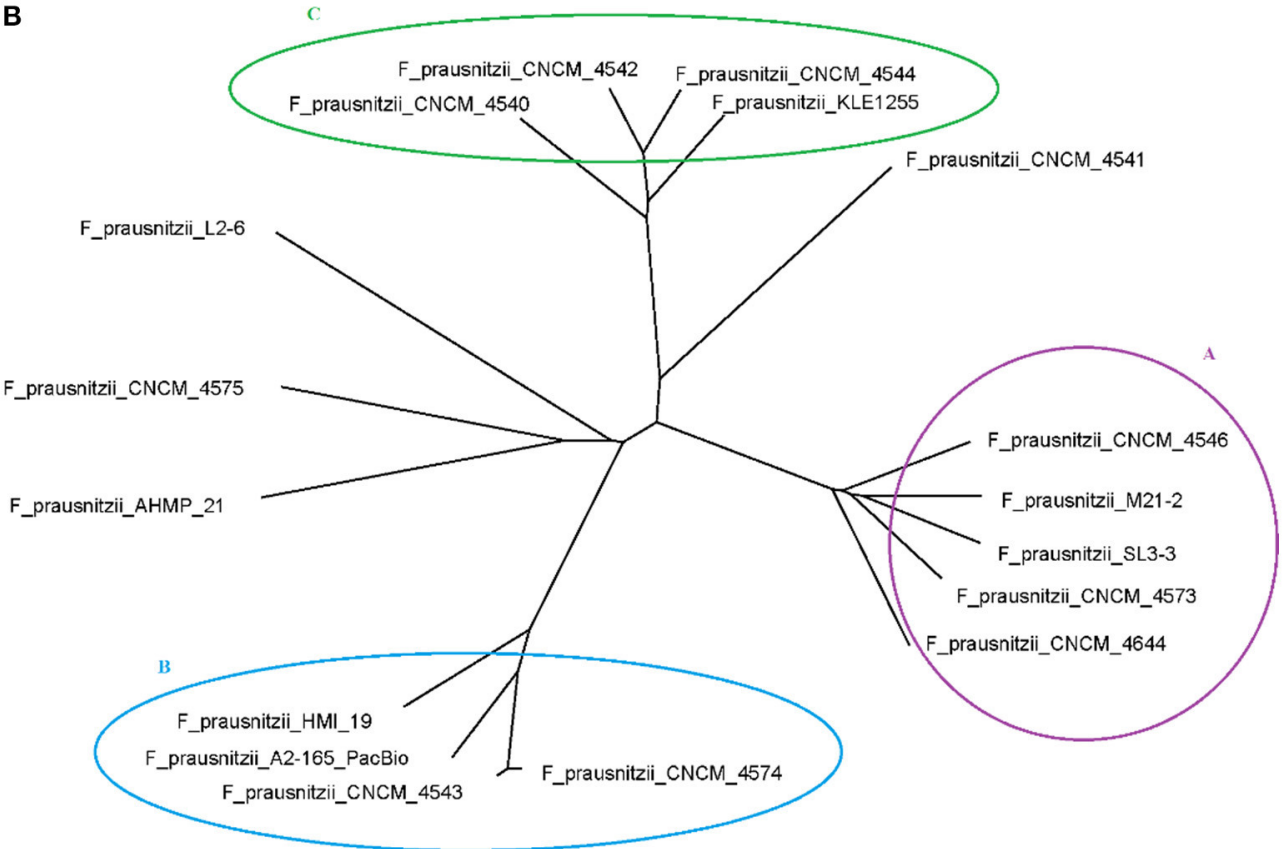

FIGURE 2 | (A) Heatmap and (B) distance-matrix-based phylogenetic network of $F$. prausnitzii. The numbers in the heatmap show the percentage of similarity between genomes; the colors vary from red (low similarity) to green (high similarity). The network was constructed using SplitsTree software with NeighborNet and equal angle methods, based on a distance matrix from Gegenees software. 
AHMP-21) did not cluster in any of the proposed groups. Likewise, the 16S rRNA sequences from three other strainsF.prausnitzii_CNCM_I-4541,F.prausnitzii_CNCM_I-4575, and F. prausnitzii_L2-6-did not cluster into any of the three groups proposed here.

The distance matrix generated using Gegenees software was plotted as a heatmap (Figure 2), in which the similarity among genomes varied from $\sim 15 \%$ (between F. prausnitzii_L2-6 and F. prausnitzii_CNCM_I_4644) to 98\% (between the genomes of $F$. prausnitzii_CNCM_I_4543 and F. prausnitzii_CNCM_I_4574, which were isolated from the same volunteer). In this analysis, the genomes of F. prausnitzii_CNCM_I-4573 and F. prausnitzii_SL3/3 clustered together with group $\mathrm{A}$, whereas the genome of F. prausnitzii_CNCM_I_4541 was only distantly related to the other strains from group C. As we found with the 16S rRNA analysis, the genome sequences of $F$. prausnitzii AHMP-21, F. prausnitzii_CNCM_I-4575, and F. prausnitzii_L2-6 did not cluster with any other sequence.

The wgMLST analysis also revealed the presence of three clusters of genomes. Once again, strain F. prausnitzii_CNCM_I_4541 was distantly related to other strains in group C, whereas strains F. prausnitzii AHMP-21, F. prausnitzii_CNCM_I-4575, and F. prausnitzii_L2-6 grouped separately from other strains (Figure 3).

\section{Average Nucleotide Identity}

We performed an Average Nucleotide Identity (ANI) analysis using whole-genome sequence data (Table 2). Using an identity cut-off of $94 \%$, this analysis also revealed the presence of the three clusters revealed by the phylogenetic analyses. In addition, the results of the ANI analysis corroborated those of the phylogenomic and wgMLST approaches in finding that the genome sequences of $F$. prausnitzii AHMP-21,
F.prausnitzii_CNCM_I-4575,F.prausnitzii_CNCM_I-4541, and F. prausnitzii_L2-6 did not cluster with any other genome sequence. As estimates of ANI are considered to be the gold standard for bacterial species determination, we used the three groups defined here for all further analyses.

\section{Gene Synteny Analysis}

Mauve software was used to order the contigs within the genomes and to identify and align regions of local collinearity (called Locally Collinear Blocks, or LCBs), which are regions without local rearrangement of probable homologous sequences that are shared by two or more genomes (Darling et al., 2004). In Figure 4, the prediction of LCBs in all strains showed small and numerous regions of homology. When the analysis considered only the genomes within a cluster, the LCBs were larger and less numerous.

\section{Pangenome Calculation}

To take a global view of the genome of Faecalibacterium and to further explore the genome diversity of this genus, we calculated the size of the pangenome (i.e., the total number of nonredundant CDSs) based on different datasets. When we examined all genomes together, the orthology analysis showed that the pangenome contained a total of 10,366 CDSs (Figure 5A), which corresponded to $\sim 3.33$-fold the average total number of genes in each of the 17 strains $(3,110.29 \mathrm{CDSs})$. When we considered only the genomes in group A, we found a total of 5,438 CDSs (Figure 5B), $\sim 1.71$-fold the average total number of CDSs in each member strain $(3,187.4)$. The pangenome of group $B$ had 4,311 CDSs (Figure 5C), which was $\sim 1.36$-fold the average total number of genes in each member strain $(3,159)$, and group $C$ had 4,686 CDSs in its pangenome (Figure 5D), which was $\sim 1.57$ fold the average total number of genes in each member strain (2,991.75). Using the formula $\alpha=1-\gamma$, we inferred that the $\alpha$

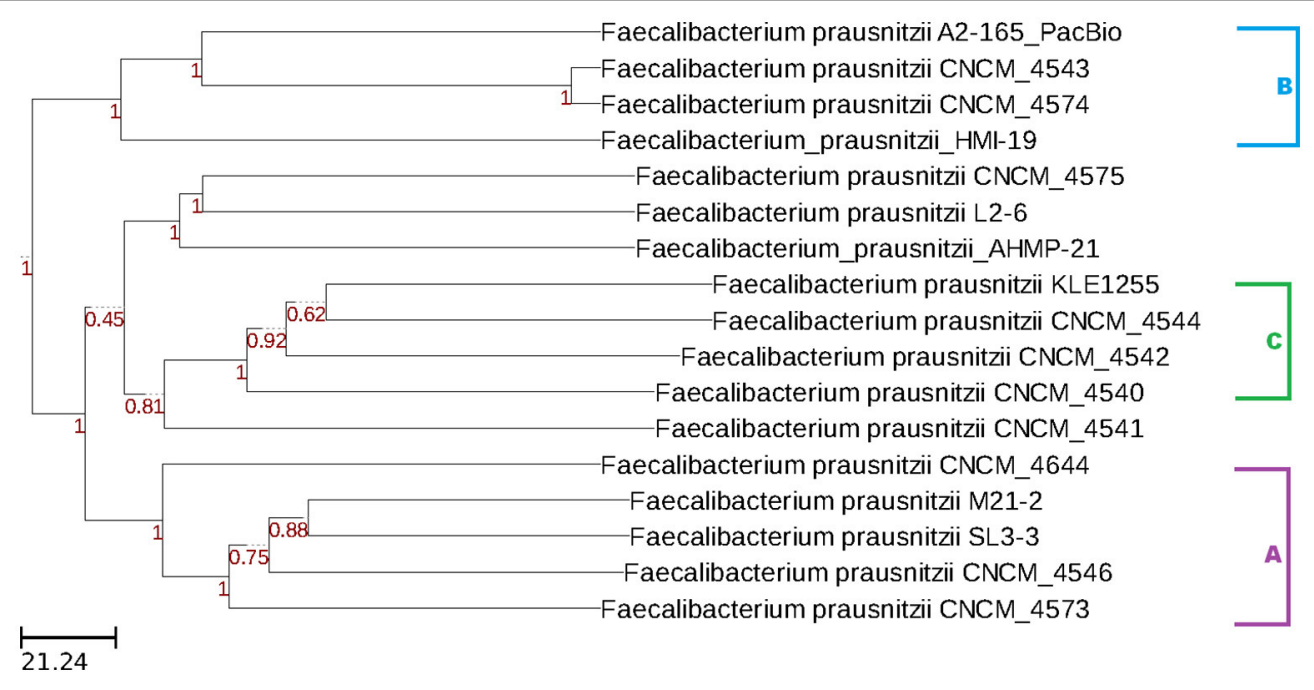

FIGURE 3 | Dendrogram constructed with wgMLST profiles for 17 F. prausnitzii genomes. The PGAdb profile from the genomes was used to construct a wgMLST tree using the Build_wgMLSTtree module (Liu et al., 2016). Bootstrap values are shown next to the nodes. The dendrogram was constructed with the UPGMA clustering algorithm. 
งิ

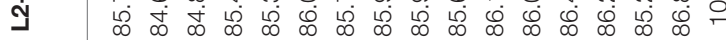

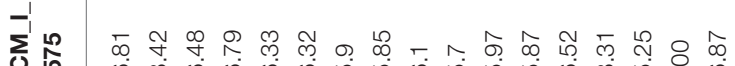

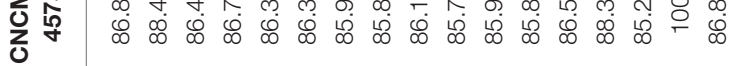

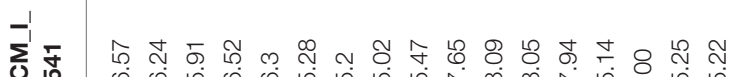

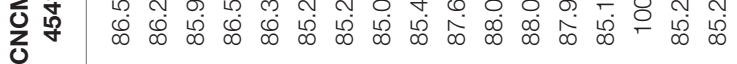
i.

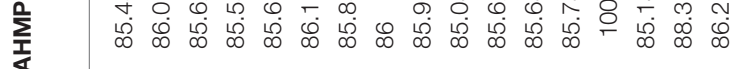

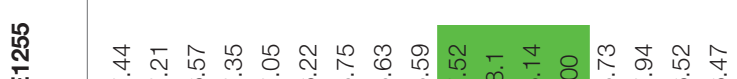

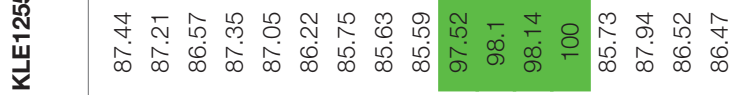
रำ

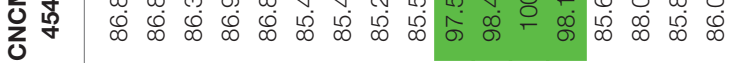
र

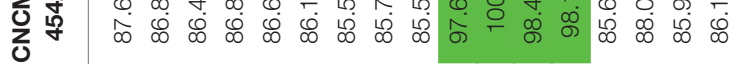

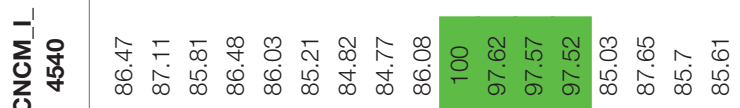

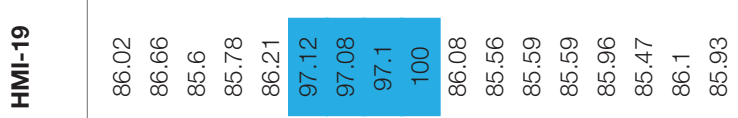
S यद

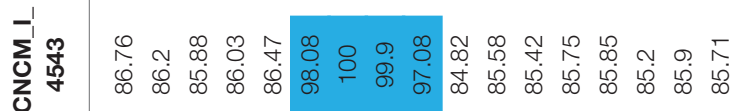

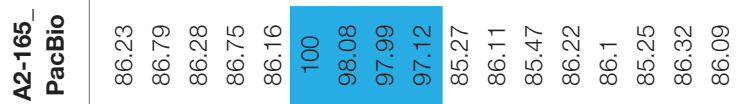
m ๘ ง

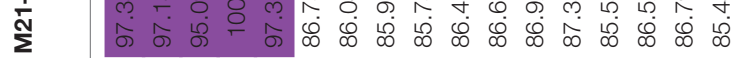

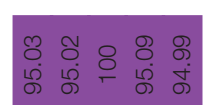

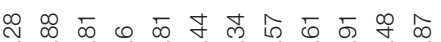

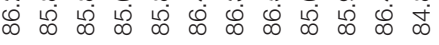

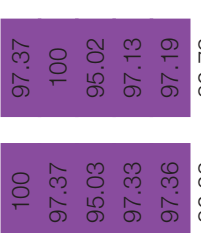

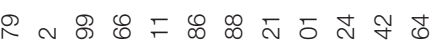

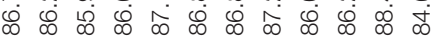
N

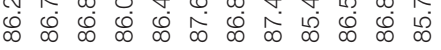

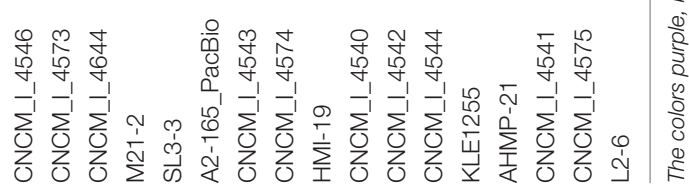

value of the pangenome of all genomes was 0.56 , indicating that the pangenome is probably open and increasing. Similarly, the extrapolation of the pangenome size calculated for groups $\mathrm{A}, \mathrm{B}$, and $\mathrm{C}$ generated $\alpha$ values of $0.63,0.77$, and 0.66 , respectively (Figure 6).

Examination of the core genome showed that 1,421 CDSs were shared by all genomes, which corresponded to less than $50 \%$ of the average gene content in each genome $(3,110.29$ CDSs) and represented $\sim 13.71 \%$ of the total pangenome. A separate analysis of the core genome of each group revealed 1,937, 2,036 , and 1,940 CDSs, respectively, in groups A, B, and C. The subset of CDSs in all genomes considered to be singletons (i.e. unique to a single genome) contained 4,465 CDSs, while withingroup analyses revealed 2,184, 988, and 1,666 singleton CDSs, respectively, within groups $\mathrm{A}, \mathrm{B}$, and $\mathrm{C}$ (Figure 5). By examining the extrapolated curve of the core genome of Faecalibacterium ssp., we found that the size of the core genome tended to converge at 1,409 genes, which represented only $13.59 \%$ of the pangenome. Within groups A, B, and C, this value increased to 1,910, 2,031, and 1,708 genes, respectively, which represented $35.12,47.11$, and $36.45 \%$ of the respective pangenome (Figure 7 ).

\section{DISCUSSION}

In bacteria, $16 \mathrm{~S}$ rRNA sequences have been widely used to study phylogenetic relationships. However, this approach is hampered by the fact that several forces that shape the evolution of bacterial genomes act with different strengths on different parts of the genome and on different bacterial lineages (Janda and Abbott, 2007; Chan et al., 2012). Therefore, to determine the diversity within a bacterial genus or species, it is important to consider not only $16 \mathrm{~S}$ rRNA sequences, but also the whole genome. Despite this, study of the evolutionary history of genus Faecalibacterium has largely been conducted through analyses of $16 \mathrm{~S}$ rRNA sequences. For example, the first study of $16 \mathrm{~S}$ rRNA gene sequences of $F$. prausnitzii revealed that this species had been misclassified into genus Fusobacterium (Wang et al., 1996; Duncan et al., 2002). After that, LopezSiles et al. (2012) used this sequence region to propose the existence of two phylogroups within what is currently considered F. prausnitzii. Furthermore, a recent study based on 16S rRNA data showed that there are significant differences among the strains of $F$. prausnitzii in resistance to antibiotics and metabolic activities (Martín et al., 2017). Here we compared the 16S rRNA gene sequences of new $F$. prausnitzii isolates to those previously available, and overall, our results challenge the current concept of a division of isolates into two broad phylogroups. As was initially proposed by Martín et al. (2017), the 16S rRNA gene sequences of our new French $F$. prausnitzii isolates can be clustered into three groups (although one, group B, was indeed supported with a lower bootstrap value than the other two). The Australian isolate F. prausnitzii_HMI-19 also clustered into group B, while the other Australian isolate, F. prausnitzii_AHMP21 , does not cluster within any of the groups proposed at present. Taken together, our analyses would suggest that there is more phylogenetic complexity in the classification of this species than has been previously shown in other studies (Wang et al., 1996; 


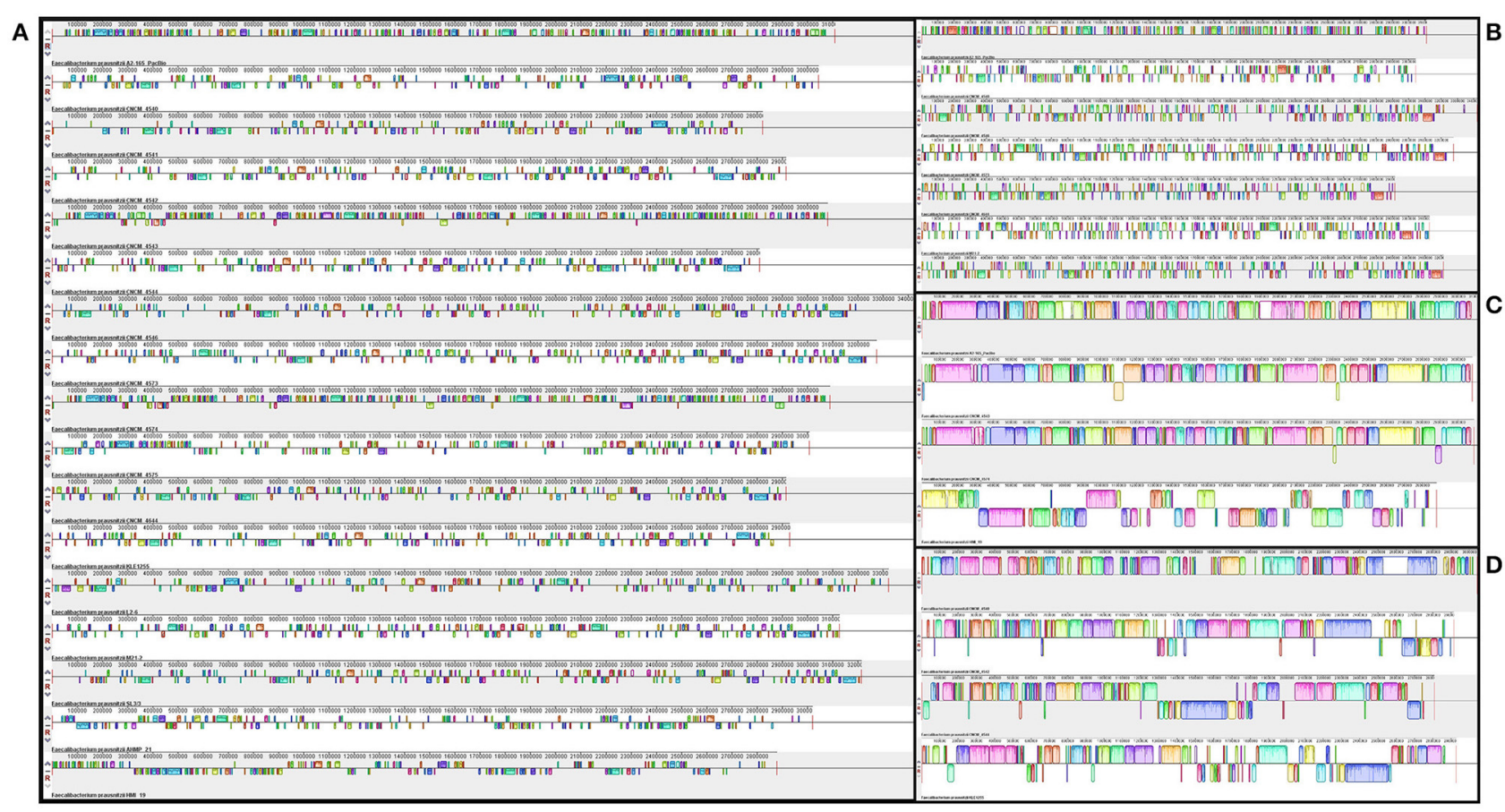

FIGURE 4 | Genomic synteny and gene conservation among the genomes of $F$. prausnitzii. The left side of the figure (A) shows the LCBs of all genomes studied here. The right side depicts the LCBs of the genomes within each of the three clusters previously obtained from ANI analysis: top right (B) - cluster A, middle right (C)-cluster B, and bottom right (D) - cluster C.

Duncan et al., 2002; Lopez-Siles et al., 2012; Martín et al., 2017). This ambiguity motivated our use of techniques other than $16 \mathrm{~S}$ analysis in order to better understand the diversity inside genus Faecalibacterium.

A whole-genome comparative analysis further validated our findings from the $16 \mathrm{~S}$ rRNA gene phylogeny. A whole-genome similarity matrix was obtained with Gegenees software and used for a phylogenomic analysis; the resulting phylogenetic tree agreed with the previously performed 16S rRNA analysis in identifying the same three groups of strains: clusters A, B, and C. In this analysis, the genospecies F.prausnitzii_CNCM_I_4541 clustered within group $\mathrm{C}$, but the relationship between this strain and other members of group $\mathrm{C}$ is very distant, reflecting the low degree of genomic similarity between the former and the latter ( $\sim 36 \%$ similarity). The same pattern was found for F. prausnitzii AHMP-21, F. prausnitzii_CNCM_I_4575, and $F$. prausnitzii_L2-6, which were grouped together, but at similarity values ranging from $\sim 23$ to $\sim 38 \%$ (as observed in the heatmap). It is interesting to note that certain strains that were isolated from the same volunteer were quite dissimilar ( 27\% similarity between FPR_CNCM_I_4573 and FPR_CNCM_I_4575; 37\% between FPR_CNCM_I_4541 and FPR_CNCM_I_4542), suggesting that the same individual may harbor different genospecies. The overall abundance of a given genospecies of Faecalibacterium within an individual host may be extremely relevant to the study of human diseases, as this overall abundance depends on the disease under study (Hippe et al., 2016; Lopez-Siles et al., 2016). For example, as part of a case-control study of atopic dermatitis (AD) in Korean subjects, Song et al. (2016) reported that 16 S rRNA PCR amplicons from stool samples of $\mathrm{AD}$ patients were enriched in those similar to strain L2-6 with respect to other strains of F. prausnitzii; they also proposed that strain L2-6 can be differentiated from other strains by the existence of a polycistronic region encoding GalNac uptake and metabolism (Song et al., 2016). Our analysis here showed that this strain does indeed demonstrate a very distinct phylogenetic pattern, which increases its potential for use as a reference strain in future $\mathrm{AD}$ studies.

To improve the resolution of our phylogenetic analysis, we also applied a strategy based on wgMLST analysis. As opposed to conventional MLST analysis, which uses only a few housekeeping genes, the wgMLST approach takes advantage of a larger number of tracked loci, enabling higher resolution in intraspecies differentiation (Maiden et al., 2013). Our analysis considered only genes that shared more than $80 \%$ coverage and $90 \%$ identity. Here, the same three groups of genospecies (A, B, and $\mathrm{C}$ ) were also detected, and again strain F. prausnitzii_CNCM_I_4541 was only distantly related to other members of group C. A group containing the isolates F. prausnitzii_AHMP-21, F. prausnitzii_CNCM_I_4575, and F. prausnitzii_L2-6 was also observed. In sum, each of the three phylogenetic analyses we performed suggested the existence of more than one genospecies within the genus Faecalibacterium. 


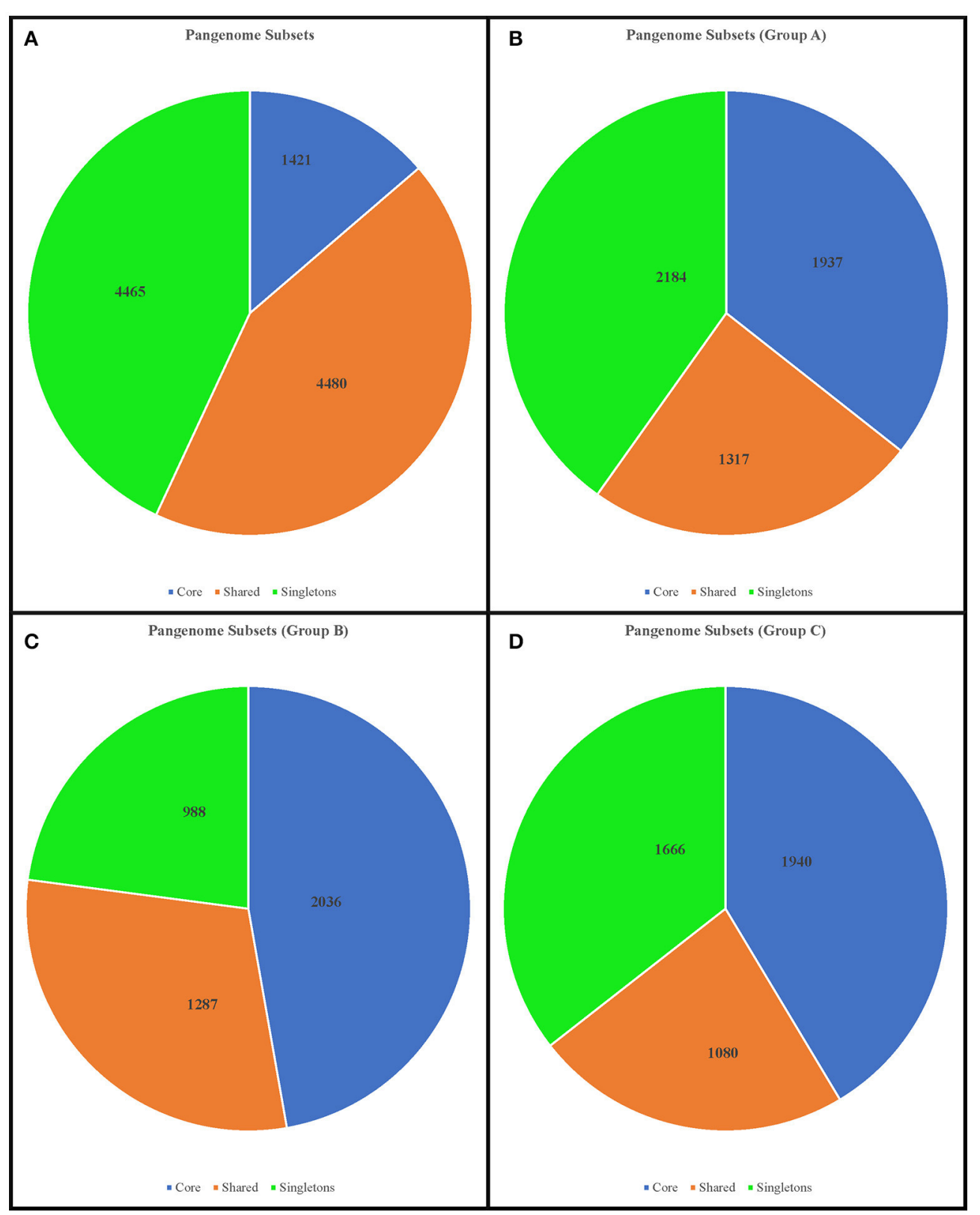

FIGURE 5 | Diagram depicting the subsets of the Faecalibacterium pangenome. The numbers represent the coding sequences belonging to each subset. Upper left chart (A): pangenome subsets from an analysis based on all 17 genomes of Faecalibacterium. Upper right chart (B): subset based on analysis of 5 genomes from group A. Lower left chart (C): subset based on analysis of 4 genomes from group B. Lower right chart (D): subset based on analysis of 4 genomes from group C.

To further corroborate the existence of these potential new "species", we performed an ANI analysis, which confirmed the new relationships identified in the previous analyses. The ANI analysis supported the classification of F. prausnitzii_CNCM_I_4541 as a distinct genospecies separate from group C; likewise, the genomes of F. prausnitzii_AHMP-21, F. prausnitzii_CNCM_I_4575, and F. prausnitzii_L2-6 were found to be quite dissimilar from all other genomes considered.

Using our revised clustering of the $F$. prausnitzii genomes, supported by the ANI results, we then investigated genome diversity via gene synteny analysis and calculations of pangenome. The extent of intra-cluster gene synteny was clearly evident in the Mauve alignments. Furthermore, the number and the lengths of the LCBs in the all-genomes dataset were strikingly different from those in the three intra-cluster datasets, which together indicated a higher degree of genome similarity within than among clusters, particularly with regard to group B. Even within a single genospecies, different genomes had a considerable number of regions with inversions and deletions, which may have arisen from horizontal gene transfer events. 


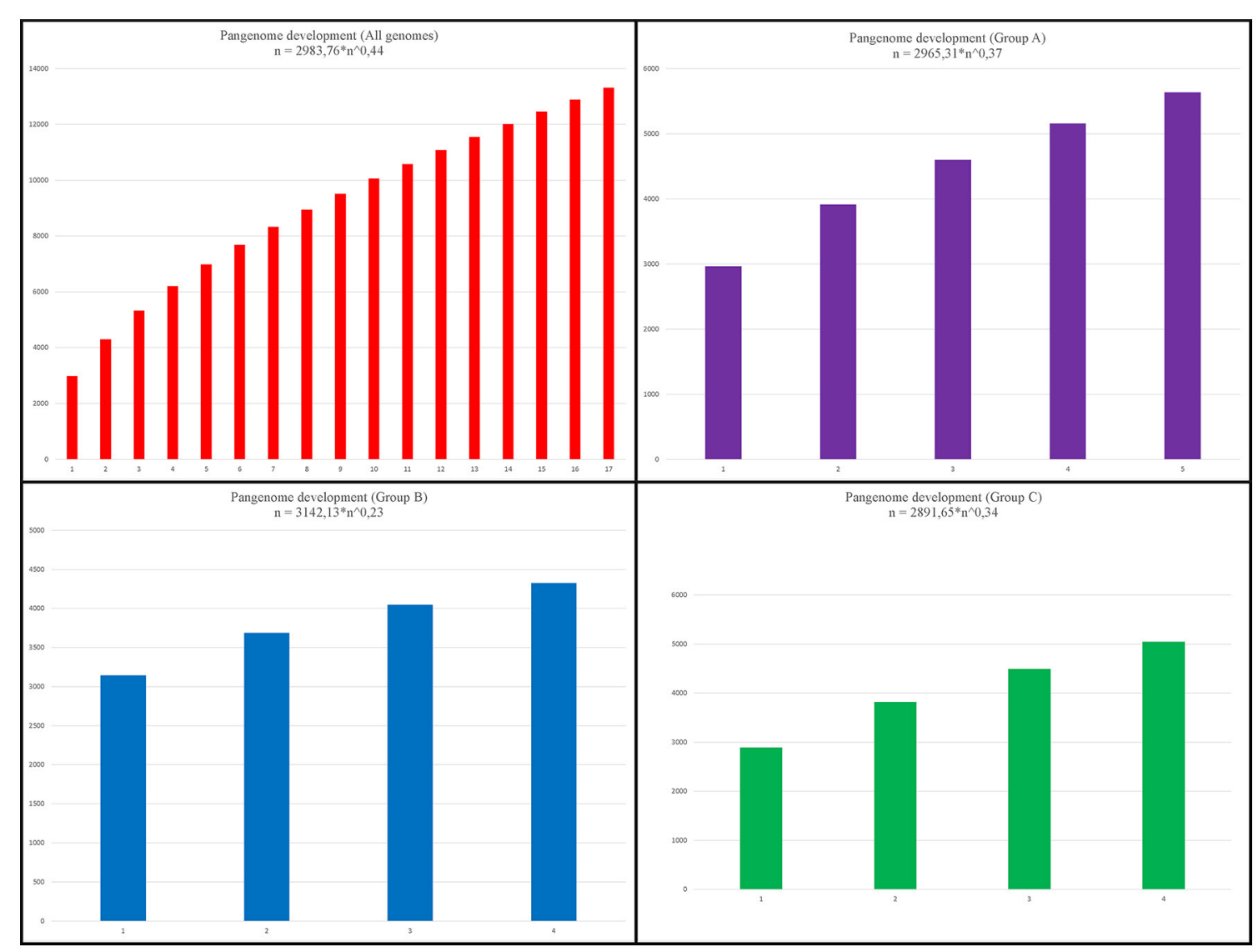

FIGURE 6 | Pangenome development. Upper left chart: pangenome development based on permutations of all 17 genomes of Faecalibacterium. Upper right chart: development based on permutations of 5 genomes from group A. Lower left chart: development based on permutations of 4 genomes from group B. Lower right chart: development based on permutations of 4 genomes from group $\mathrm{C}$.

Again, though, the genomes from group B were more similar to each other than were the genomes of the other groups.

The same four datasets were used to perform calculations of pangenome. As might be expected, the number of core-genome CDSs was greater within each cluster than within the dataset containing all 17 genomes, which is consistent with the idea that the genomes within a cluster are from the same species. Extrapolations of pangenome development also corroborated this assumption. The $\alpha$ value generated from an analysis of all genomes indicated that the genus Faecalibacterium has an open pangenome ( $\alpha=0.56$ ), as does each of the groups ( $\alpha=0.63, \alpha$ $=0.77$, and $\alpha=0.66$, respectively). However, the intra-group $\alpha$ values reveal that these latter pangenomes are increasing in size more slowly than the pangenome of all species (as indicated by higher $\alpha$ values). This means that, if we consider all the genomes to be part of the genus Faecalibacterium, each new genome sequenced will increase substantially the number of nonredundant genes in this genus. On the other hand, the genomes within each group tend to be more clonal, and newly sequenced genomes included within these groups will have a less prominent effect on the number of non-redundant genes. We likewise arrived at the same conclusion by analyzing the development of the core genome and singletons: the final core genome tended to be larger within each genospecies than within the all-genome analysis. This phylogenetic approach to pangenome analysis revealed patterns that were totally in accordance with the results of our other analyses.

\section{CONCLUDING REMARKS}

Here, we used a variety of methods to analyze 16S rRNA and whole genome data, which together showed that: (i) the current application of phylogroups to differentiate among strains of F. prausnitzii should be revised; (ii) this genus contains at least three separate clusters, spanning both phylogroups I and II, which are all derived from a common recent ancestor; and (iii) some strains (e.g., F. prausnitzii AHMP-21, F. prausnitzii_L2-6, and F. prausnitzii_CNCM_I_4575) appear to represent a deeper, more divergent branch of "Faecalibacterium prausnitzii." Collectively, our results provide evidence for the reconsideration of the phylogenetic and genomic relatedness among strains currently assigned to $F$. prausnitzii. In addition, they highlight the need for lineage (strain-based) differentiation within this genus to better define how specific members might 


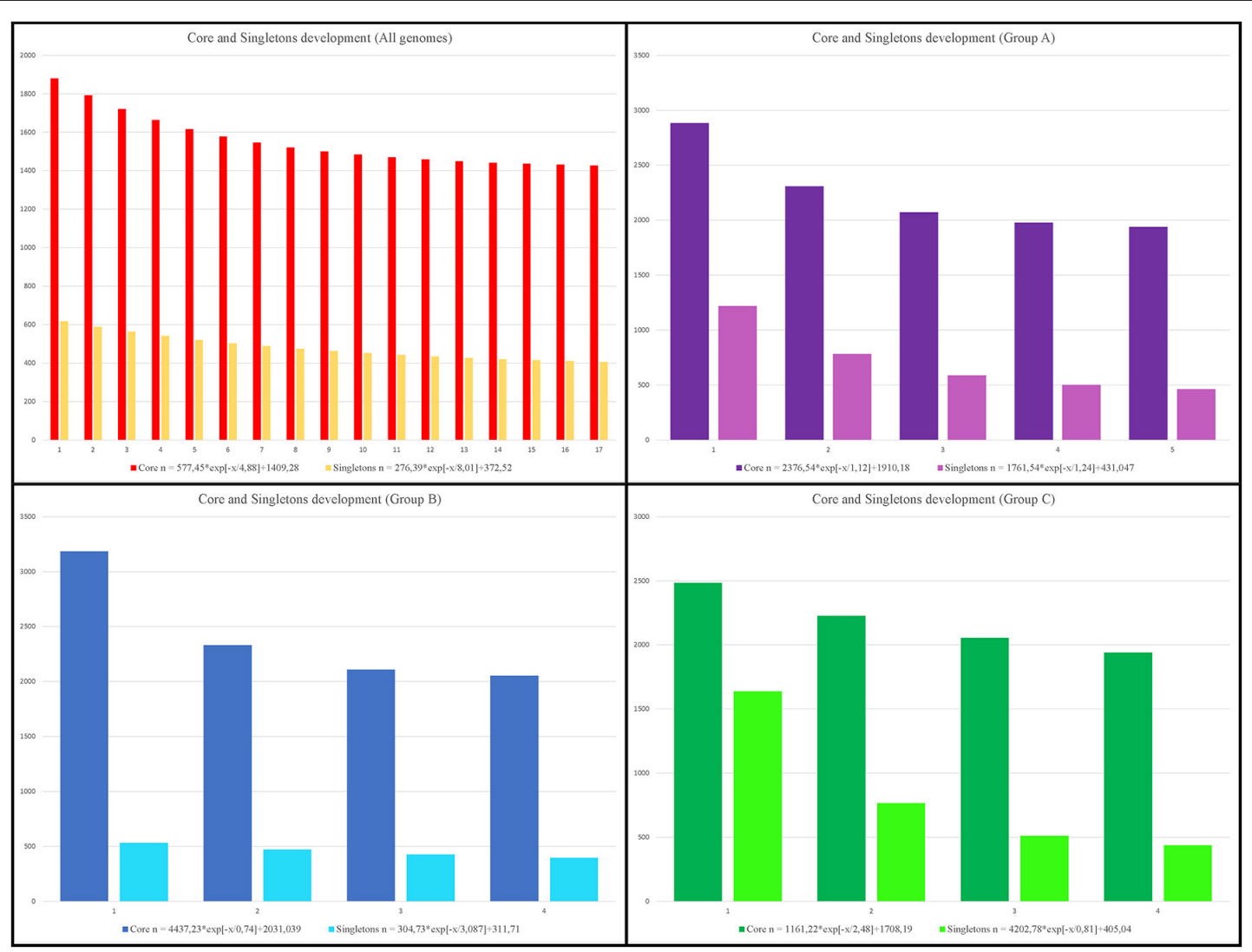

FIGURE 7 | Development of core genome and singletons. Upper left chart: core-genome and singleton development based on permutations of all 17 genomes of Faecalibacterium. Upper right chart: development based on permutations of 5 genomes from group A. Lower left chart: development based on permutations of 4 genomes from group B. Lower right chart: development based on permutations of 4 genomes from group C.

be associated with positive or negative host interactions. Such lineage-specific variations might not only explain the variable abundances of $F$. prausnitzii linked with adverse health outcomes (e.g., atopic dermatitis, Crohn's disease, and ulcerative colitis; Swidsinski et al., 2008; Hansen et al., 2012), but also provide new opportunities for the diagnosis and strain-specific treatment of gut inflammation and associated diseases. Also, to the best of our knowledge, this is the first work to combine an analysis of pangenome development with ANI analysis in order to corroborate the assignment of strains to new species. Here, we propose that pangenome subsets and the $\alpha$ value generated by these analyses may be used as an alternative approach, together with ANI, for the in silico classification of new species. Although low $\alpha$ values may be found inside a species cluster, due to a high degree of variation among genomes arising from intense horizontal gene transfer events, a high intra-cluster $\alpha$ value may be considered a good indicator of a new, more-clonal species inside the genus.

\section{AUTHOR CONTRIBUTIONS}

LB, SB, RM, VR, MT, SM, FC, HS, LGB, MM, PL, VA, JC, and SS designed the experiments, revised the manuscript critically, and participated in the design of the project. LB, SB, RM, VR, SM,
FC, and SC performed the experiments and analysis. LB and SC drafted the manuscript.

\section{FUNDING}

This work was supported by a scholarship from the International Cooperation Program CAPES/COFECUB (financed by the Brazilian Federal Agency for Support and Evaluation of Graduate Education within the Ministry of Education of Brazil) and was conducted at the "Institut National de la Recherche Agronomique" (INRA), France. This paper was a part of FPARIS collaborative project selected and supported by the Vitagora Competitive Cluster and funded by the French FUI (Fond Unique Interministériel; FUI: $\left.\mathrm{n}^{\circ} \mathrm{F} 1010012 \mathrm{D}\right)$, FEDER (Fonds Européen de Développement Régional; Bourgogne: 34606), the region of Burgundy, the Conseil Général 21, and the Grand Dijon. This work was also supported by Merck Médication Familiale (Dijon, France) and Biovitis (Saint Etienne de Chomeil, France). RM and SM receive a salary from these same grants. $\mathrm{SB}$ is the recipient of an Australian Postgraduate award. The contributions of MM and SB have been partially supported with funds from the University of Queensland, the University of Queensland Diamantina Institute, and the Helmsley Charitable Trust (via the Australasian Gastro Intestinal Foundation). 


\section{REFERENCES}

Agren, J., Sundström, A., Håfström, T., and Segerman, B. (2012). Gegenees: fragmented alignment of multiple genomes for determining phylogenomic distances and genetic signatures unique for specified target groups. PLOS ONE 7:e39107. doi: 10.1371/journal.pone.0039107

Aziz, R. K., Bartels, D., Best, A. A., DeJongh, M., Disz, T., Edwards, R. A., et al. (2008). The RAST Server: rapid annotations using subsystems technology. BMC Genomics 9:75. doi: 10.1186/1471-2164-9-75

Castillo, M., Skene, G., Roca, M., Anguita, M., Badiola, I., Duncan, S. H., et al. (2007). Application of $16 \mathrm{~S}$ rRNA gene-targetted fluorescence in situ hybridization and restriction fragment length polymorphism to study porcine microbiota along the gastrointestinal tract in response to different sources of dietary fibre. FEMS Microbiol. Ecol. 59, 138-146. doi: 10.1111/j.1574-6941.2006.00204.x

Chan, J. Z. M., Halachev, M. R., Loman, N. J., Constantinidou, C., and Pallen, M. J. (2012). Defining bacterial species in the genomic era: insights from the genus Acinetobacter. BMC Microbiol. 12:302. doi: 10.1186/1471-2180-12-302

Darling, A. C. E., Mau, B., Blattner, F. R., and Perna, N. T. (2004). Mauve: multiple alignment of conserved genomic sequence with rearrangements. Genome Res. 14, 1394-1403. doi: 10.1101/gr.2289704

Duncan, S. H., Hold, G. L., Harmsen, H. J. M., Stewart, C. S., and Flint, H. J. (2002). Growth requirements and fermentation products of Fusobacterium prausnitzii, and a proposal to reclassify it as Faecalibacterium prausnitzii gen. nov., comb. nov. Int. J. Syst. Evol. Microbiol. 52, 2141-2146. doi: 10.1099/00207713-52-6-2141

Gurevich, A., Saveliev, V., Vyahhi, N., and Tesler, G. (2013). QUAST: quality assessment tool for genome assemblies. Bioinformatics 29, 1072-1075. doi: 10.1093/bioinformatics/btt086

Hansen, R., Russell, R. K., Reiff, C., Louis, P., McIntosh, F., Berry, S. H., et al. (2012). Microbiota of de-novo pediatric ibd: increased Faecalibacterium prausnitzii and reduced bacterial diversity in Crohn's but not in ulcerative colitis. Am. J. Gastroenterol. 107, 1913-1922. doi: 10.1038/ajg.2012.335

Hippe, B., Remely, M., Aumueller, E., Pointner, A., Magnet, U., and Haslberger, A. G. (2016). Faecalibacterium prausnitzii phylotypes in type two diabetic, obese, and lean control subjects. Benef. Microbes 7, 511-517. doi: 10.3920/BM2015.0075

Huson, D. H. (2005). Application of phylogenetic networks in evolutionary studies. Mol. Biol. Evol. 23, 254-267. doi: 10.1093/molbev/msj030

Janda, J. M., and Abbott, S. L. (2007). 16S rRNA gene sequencing for bacterial identification in the diagnostic laboratory: pluses, perils, and pitfalls. J. Clin. Microbiol. 45, 2761-2764. doi: 10.1128/JCM. 01228-07

Kim, M., Oh, H. S., Park, S. C., and Chun, J. (2014). Towards a taxonomic coherence between average nucleotide identity and 16S rRNA gene sequence similarity for species demarcation of prokaryotes. Int. J. Syst. Evol. Microbiol. 64, 346-351. doi: 10.1099/ijs.0. 059774-0

Kimura, M. (1980). A simple method for estimating evolutionary rates of base substitutions through comparative studies of nucleotide sequences. J. Mol. Evol. 16, 111-120. doi: 10.1007/BF01731581

Konstantinidis, K. T., and Tiedje, J. M. (2005). Genomic insights that advance the species definition for prokaryotes. Proc. Natl. Acad. Sci. U.S.A. 102, 2567-2572. doi: 10.1073/pnas.04097 27102

Kumar, S., Stecher, G., and Tamura, K. (2016). MEGA7: molecular evolutionary genetics analysis version 7.0 for bigger datasets. Mol. Biol. Evol. 33, 1870-1874. doi: $10.1093 / \mathrm{molbev} / \mathrm{msw} 054$

Li, L., Stoeckert, C. J., and Roos, D. S. (2003). OrthoMCL: identification of ortholog groups for eukaryotic genomes. Genome Res. 13, 2178-2189. doi: $10.1101 /$ gr. 1224503

Liu, Y. Y., Chiou, C. S., and Chen, C. C. (2016). PGAdb-builder: a web service tool for creating pan-genome allele database for molecular fine typing. Sci. Rep. 6:36213. doi: $10.1038 / \mathrm{srep} 36213$

Lopez-Siles, M., Duncan, S. H., Garcia-Gil, L. J., and Martinez-Medina, M. (2017). Faecalibacterium prausnitzii: from microbiology to diagnostics and prognostics. ISME J. 11, 841-852. doi: 10.1038/ismej.2016.176
Lopez-Siles, M., Khan, T. M., Duncan, S. H., Harmsen, H. J. M., GarciaGil, L. J., and Flint, H. J. (2012). Cultured representatives of two major phylogroups of human colonic Faecalibacterium prausnitzii can utilize pectin, uronic acids, and host-derived substrates for growth. Appl. Environ. Microbiol. 78, 420-428. doi: 10.1128/AEM. 06858-11

Lopez-Siles, M., Martinez-Medina, M., Surís-Valls, R., Aldeguer, X., SabatMir, M., Duncan, S. H., et al. (2016). Changes in the abundance of Faecalibacterium prausnitzii phylogroups I and II in the intestinal mucosa of inflammatory bowel disease and patients with colorectal cancer. Inflamm. Bowel Dis. 22, 28-41. doi: 10.1097/MIB.0000000000 000590

Maiden, M. C. J., van Rensburg, M. J. J., Bray, J. E., Earle, S. G., Ford, S. A., Jolley, K. A., et al. (2013). MLST revisited: the gene-by-gene approach to bacterial genomics. Nat. Rev. Microbiol. 11, 728-736. doi: 10.1038/ nrmicro3093

Martín, R., Miquel, S., Benevides, L., Bridonneau, C., Robert, V., Hudault, S., et al. (2017). Functional characterization of novel Faecalibacterium prausnitzii strains isolated from healthy volunteers: a step forward in the use of $F$. prausnitzii as a next-generation probiotic. Front. Microbiol. 8:1226. doi: 10.3389/fmicb.2017. 01226

Miquel, S., Martín, R., Bridonneau, C., Robert, V., Sokol, H., Bermúdez-Humarán, L. G., et al. (2014). Ecology and metabolism of the beneficial intestinal commensal bacterium Faecalibacterium prausnitzii. Gut Microb. 5, 146-151. doi: 10.4161/gmic. 27651

Miquel, S., Martín, R., Rossi, O., Bermúdez-Humarán, L. G., Chatel, J. M., Sokol, H., et al. (2013). Faecalibacterium prausnitzii and human intestinal health. Curr. Opin. Microbiol. 16, 255-261. doi: 10.1016/j.mib.2013. 06.003

Nava, G. M., and Stappenbeck, T. S. (2011). Diversity of the autochthonous colonic microbiota. Gut Microbes 2, 99-104. doi: 10.4161/gmic.2.2. 15416

Oikonomou, G., Teixeira, A. G. V., Foditsch, C., Bicalho, M. L., Machado, V. S., and Bicalho, R. C. (2013). Fecal microbial diversity in pre-weaned dairy calves as described by pyrosequencing of metagenomic $16 \mathrm{~S}$ rDNA. Associations of Faecalibacterium species with health and growth. PLoS ONE 8:e63157. doi: 10.1371/journal.pone. 0063157

Rajilić-Stojanović, M., Biagi, E., Heilig, H. G. H. J., Kajander, K., Kekkonen, R. A., Tims, S., et al. (2011). Global and deep molecular analysis of microbiota signatures in fecal samples from patients with irritable bowel syndrome. Gastroenterology 141, 1792-1801. doi: 10.1053/j.gastro.2011. 07.043

Scupham, A. J. (2007). Succession in the intestinal microbiota of preadolescent turkeys. FEMS Microbiol. Ecol. 60, 136-147. doi: 10.1111/j.1574-6941.2006. 00245. $\mathrm{x}$

Sokol, H., Pigneur, B., Watterlot, L., Lakhdari, O., BermudezHumaran, L. G., Gratadoux, J. J., et al. (2008). Faecalibacterium prausnitzii is an anti-inflammatory commensal bacterium identified by gut microbiota analysis of Crohn disease patients. Proc. Natl. Acad. Sci. U.S.A. 105, 16731-16736. doi: 10.1073/pnas.0804 812105

Song, H., Yoo, Y., Hwang, J., Na, Y.-C., and Kim, H. S. (2016). Faecalibacterium prausnitzii subspecies-level dysbiosis in the human gut microbiome underlying atopic dermatitis. J. Allergy Clin. Immunol. 137, 852-860. doi: 10.1016/j.jaci.2015.08.021

Swidsinski, A., Loening-Baucke, V., Vaneechoutte, M., and Doerffel, Y. (2008). Active Crohn's disease and ulcerative colitis can be specifically diagnosed and monitored based on the biostructure of the fecal flora. Inflamm. Bowel Dis. 14, 147-161. doi: 10.1002/ibd. 20330

Tap, J., Mondot, S., Levenez, F., Pelletier, E., Caron, C., Furet, J.-P., et al. (2009). Towards the human intestinal microbiota phylogenetic core. Environ. Microbiol. 11, 2574-2584. doi: 10.1111/j.1462-2920.2009. 01982.x 
Thompson, J. D., Higgins, D. G., and Gibson, T. J. (1994). CLUSTAL W: improving the sensitivity of progressive multiple sequence alignment through sequence weighting, position-specific gap penalties and weight matrix choice. Nucleic Acids Res. 22, 4673-4680. doi: 10.1093/nar/22. 22.4673

Walker, A. W., Ince, J., Duncan, S. H., Webster, L. M., Holtrop, G., Ze, X., et al. (2011). Dominant and diet-responsive groups of bacteria within the human colonic microbiota. ISME J. 5, 220-230. doi: 10.1038/ismej0.2010.118

Wang, R. F., Cao, W. W., and Cerniglia, C. E. (1996). Phylogenetic analysis of Fusobacterium prausnitzii based upon the 16S rRNA gene sequence and PCR confirmation. Int. J. Syst. Bacteriol. 46, 341-343. doi: 10.1099/00207713-46-1-341
Conflict of Interest Statement: The authors declare that the research was conducted in the absence of any commercial or financial relationships that could be construed as a potential conflict of interest.

Copyright (๔) 2017 Benevides, Burman, Martin, Robert, Thomas, Miquel, Chain, Sokol, Bermudez-Humaran, Morrison, Langella, Azevedo, Chatel and Soares. This is an open-access article distributed under the terms of the Creative Commons Attribution License (CC BY). The use, distribution or reproduction in other forums is permitted, provided the original author(s) or licensor are credited and that the original publication in this journal is cited, in accordance with accepted academic practice. No use, distribution or reproduction is permitted which does not comply with these terms. 